
\title{
$\begin{array}{ll}\text { Research Square } & \begin{array}{l}\text { Preprints are preliminary reports that have not undergone peer review. } \\ \text { They should not be considered conclusive, used to inform clinical practice, } \\ \text { or referenced by the media as validated information. }\end{array}\end{array}$
}

\section{Using lipidomics to reveal details of lipid accumulation in developing Siberian apricot (Prunus sibirica L.) seed kernels}

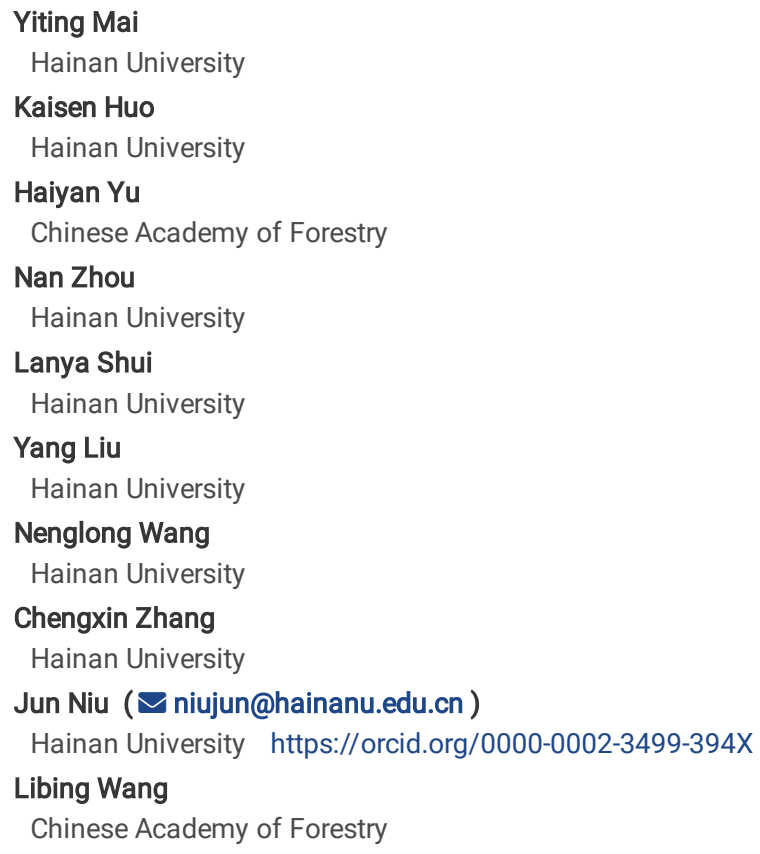

Version of Record: A version of this preprint was published at GCB Bioenergy on May 22nd, 2020. See the published version at https://doi.org/10.1111/gcbb.12693. 


\section{Abstract}

Background: The oil content of Siberian apricot seed kernel (SASK) is generally as high as $50 \%$, and biodiesel fuel properties of SASK oil were conformed to EN 14214 and GB/T 20828-2007 standards. Thus, Prunus sibirica is a novel non-crop feedstock for biodiesel production in China. We have been addressing this issue by studying the regulation of oil accumulation in SASK.

Results: As part of this research we have carried out a detailed lipidomic analysis in developing SASK. Here, oil contents and fatty acid (FA) compositions were studied in developing SASK from AS-80 and AS-84, at intervals of 1 week from 3 weeks after anthesis (WAA) to 9 weeks. The major differences between the two germplasms are higher contents of C18:1 and C18:2 in AS-80 than in AS-84 at a mature stage. The SASKs of 4, 6 and 8 WAA, respectively representing early, mid and late phases of oil accumulation, were selected as optimal samples for lipidomic analysis. The molecular species in individual lipid classes between AS-80 and AS-84 were similar, and exhibited quite distinct patterns with SASK development. Additionally, the lipidomic data coupled with qRT-PCR analysis suggested that three mechanisms allow the flux of FA through phosphatidylcholine that influenced the molecular composition of eventual TAG.

Conclusions: The first report on lipidomic analysis during seed development in wood oilseed plants. Our data contribute significantly to understand the underlying mechanisms of lipid accumulation in P. sibirica, and may also present strategies for engineering oil accumulation in oilseed plants.

\section{Background}

Siberian apricot (Prunus sibirica) is a woody oil species that belongs to the Rosaceae family. As an endemic species in northern China, it is widely used for soil and water conservation owing to its superior adaptability to a wide range of environment [1]. Almost 192,500 tonnes of seeds are harvested in autumn every year [2]. The oil content of Siberian apricot seed kernel (SASK) is generally as high as $50 \%$, of which about $95 \%$ is unsaturated fatty acid (FA), including 56.23-76.69\% oleic acid and 16.44-34.69\% linoleic acid [3]. Because the cost of feedstock account for the cost of biodiesel approximately over 75\% [4], noncrop feedstock to produce biodiesel has drawn governmental and popular attention. Previous evaluation of SASK oil has shown that its biodiesel fuel properties, such as cetane number, iodine number and oxidation stability, were conformed to EN 14214 and GB/T 20828-2007 standards [3, 5]. Thus, SASK oil is a potential non-crop feedstock for biodiesel production.

In plants, sucrose can be transported from photosynthetic tissues to heterotrophic sinks through the phloem. Once delivered into sinks, sucrose can be cleaved into two hexoses by either invertase or sucrose synthase [6]. In oil seeds, the generated hexoses are metabolized through the oxidative pentose phosphate pathway and the glycolytic pathway, providing acetyl-CoA as precursor for de novo FA synthesis [7]. After FA formation in plastid, a mixture of palmitic acid (C16:0), palmitoleic acid (C16:1), stearic acid (C18:0) and oleic acid (C18:1) is produced, and transported to cytosolic acyl-CoA pool [8]. These long-chain acylCoAs are the substrate for glycerolipid assembly (Kennedy pathway). Notably, while the classic Kennedy pathway relies on the sequential acylation of a glycerol-3-phosphate (G3P) backbone, the membrane lipid phosphatidylcholine (PC) is also important for the assembly of unsaturated or otherwise modified lipid in endoplasmic reticulum [9]. Three mechanisms allow the flux of FA through PC for eventual triacylglycerol (TAG) synthesis. First, acyl-

CoA:lysophosphatidylcholine acyltransferase catalyzes the esterification of nascent FAs to PC via "Acyl editing" [10]. Second, PC-derived diacylglycerol (DAG) is utilized as the substrate for TAG synthesis [9]. Third, the FA from PC pool can be esterified to sn-3 position of TAG by phospholipid:DAG acyltransferase (PDAT) [11]. Overall, PC pool is a central intermediate in flux of acyl chains, implying that lipid assembly exerted more control over oil accumulation than FA biosynthesis.

Although the basic characteristics of functional enzymes involved in the biochemical pathways of oil accumulation have been well understood in model species Arabidopsis [7, 11], less is known about the regulatory mechanism of oil accumulation in wood oil plants. Previously, we have studied the regulation of oil accumulation in developing SASK using high-throughput sequencing, revealing the involvement of some specific genes encoding functional enzymes and transcription factors in SASK oil accumulation [12-14]. However, some detailed controls could not be delineated. For example, why have phosphatidylcholine: diacylglycerol cholinephosphotransferase (PDCT) and diacylglycerol cholinephosphotransferase (DAG-CPT) higher expression levels in early development of SASK [12]? Moreover, the relative flux of acyl chains onto the sn-3 position of TAG through either PDAT or diacylglycerol acyltransferase (DGAT) is unclear in most oilseed plants [8]. Increasing studies in Arabidopsis elucidated overlapping and complementary functions of DGAT and PDAT [15, 16]. A double gene silencing reduced oil $80 \%$ in Arabidopsis seeds [17]. However, the transcriptional level and enzyme activity for DGAT were much higher than that for PDAT in oilseed rape $[18,19]$, implying a more important role of DGAT in TAG assembly. During developing SASK, DGAT1 and PDAT2 were identified with the highest expression value at middle and late development, respectively, suggesting an alteration of TAG assembly by PDAT2 though PC pool or by DGAT1 via Kennedy pathway [12].

Lipidomics as a novel technique can provide important data about lipid metabolism during oil accumulation. Compared with other metabolites, the composition and content of lipids could reflect the response of plants to specific stimuli. Lipometabolism is the master pathway for energy homeostasis, membrane structure, cell signaling, transcription and translation regulation, and cell interactions. Recent studies have indicated the importance of lipids in response to biotic and abiotic stresses [20,21]. Moreover, lipidomics technique has been applied to various plant tissues [22-24]. To identify the optimal period of SASK used for lipidomic analysis, the oil contents and FA compositions from two different germplasms (AS-85 and AS-86) were first detected. Subsequently, we applied liquid chromatography-tandem mass spectrometry (LC-MS/MS) to developing SASK. The lipidomic data could provide detailed information about the end products and the intermediates of glycerolipid metabolism. The expression levels of some key genes were assessed using quantitative reverse transcription PCR (qRT-PCR). This temporal and spatial regulation model of oil accumulation in developing SASK provides a basis for improving our understanding of TAG synthesis in P. sibirica, and may also provide some reference for researching the wood oil plants.

\section{Results}




\section{Dynamic changes of oil content and FA compositions in developing SASK}

The oil accumulation during SASK development showed a sigmoid pattern, exhibiting a sharp rise between 4 and 8 WAA and a maximal level 8 WAA (Figure 1). Comparative analysis indicated that the oil increase was higher in AS-80 than in AS-84, which ultimately led to a higher oil content in AS-80 ( 53.48 $\mathrm{g} / 100 \mathrm{~g}$ ) than in AS-84 (44.85 g/100 g). GC-MS analysis indicated that eight FA species are predominantly found in SASKs, namely 16:0, 16:1, 18:0, 18:1, 18:2 and 18:3 (polyunsaturated fatty acids, PUFAs), 20:0 and 20:1(very long-chain fatty acids, VLCFAs; C冈20) (Table 1). Among those FAs, C18:1 (oleic acid) and C18:2 (linoleic acid) represented the major compositions in SASK oil (Table 1). The FA profiles were different between AS-80 and AS-84, and the former exhibited a higher content of oleic acid and linoleic acid (Table 1). Based on these results, the SASKs from 4, 6 and 8 WAA, which respectively represent early, mid and late phases of oil accumulation, were selected as optimal samples for lipidomic analysis.

\section{Changes to TAG molecular species during SASK development}

Triglycerides, a naturally occurring ester of three FAs and glycerol, are the chief constituent of storage oil. After identifying the TAG molecular species, the two prominent species were 18:1/18:1/18:2 and 18:1/18:1/18:3, followed by 18:2/18:2/18:2, 18:1/18:1/18:1, 16:1/18:1/18:2 (Supplementary Table S1 and Figure 2). It is worth noticing that C18:1 and C18:2 are the main FA chains at all time points, in keeping with the analytic results of FA compositions (Table 1). Although these TAG molecules both in AS-80 and AS-84 showed a gradual accumulation during development, a significantly increased content (especially for 18:1/18:2/18:2 and 18:1/18:1/18:3) could be observed in AS-80 (Figure 2). The different rate of increment could explain why the SASKs from AS-80 have a higher oil content. It will be noted that those TAG molecules containing VLCFAs are only minor components (Supplementary Table S1).

\section{Changes to DAG and PA molecules during SASK development}

The main molecular species of DAG in developing SASK were 18:3/18:3, 18:1/18:1 and 18:2/18:2 (Figure 3A). The content of 18:3/18:3 showed notable decrease during oil accumulation, whereas 18:1/18:1 and 18:2/18:2 were little changed throughout development (Figure 3A and Supplementary Table S2). There were only very small amounts of DAG molecular species containing VLCFAs (Supplementary Table S2).

After removing the phosphate group, PA molecules can be invoked as the immediate precursor to DAG in the Kennedy pathway. The main molecular species of PA were 18:1/18:1 and 16:0/18:2 (Figure 3B and Supplementary Table S3). This was particularly noticeable with similarly dynamic profiles between AS-80 and AS-84. The 18:1/18:1 increase in abundance between 4 and 6 WAA, while 16:0/18:2 decreased with SASK maturity (Figure 3B). Other significant molecular species of PA were those containing PUFAs (18:1/18:2, 16:0/18:3, 18:2/18:1, 18:3/18:3, 18:2/18:3 and 18:3/18:2) or those containing VLCFAs (18:1/20:1 and 18:1/20:2). During SASK development, most of those PA species containing PUFAs and VLCFAs tended to decrease (Supplementary Table S3).

\section{Changes to phospholipids during SASK development}

In plants, PC is a central intermediate in the flux of FAs or DAGs, or both substrates into TAG. By lipidomic analysis, the two main molecular species in developing SASK were 18:1/18:1 and 18:1/18:2, and both showed a significant rise with the development of SASK (Figure 4A and Supplementary Table S4). Other significant PC molecular species were 16:0/18:1, 18:2/18:1, 16:0/18:3, 16:1/16:1, 18:3/18:3 and 18:2/18:2 (Figure 4A). Interestingly, those PC species containing PUFAs had the maximum values at 6 WAA (the mid stage of oil accumulation). There were only minor amounts of PC species containing VLCFAs (Supplementary Table S4).

Phosphatidyl-ethanolamine (PE) is synthesized using the same enzyme with PE biosynthesis and DAG from the Kennedy pathway. Here, we examined PE molecular species to compare with those of PC. The main molecular species of PE were 16:0/18:3, 18:1/18:1, 16:0/18:1 and 18:2/18:2 (Figure 4B and Supplementary Table S5). It was clear from the data that the 18:2/18:2 accumulation during SASK development showed a V-pattern with a minimum value at 6 WAA, whereas 16:0/18:3, 18:1/18:1 and 16:0/18:1 represented an inverse pattern with a maximum value at 6 WAA (Figure 4B). The temporal pattern of PE molecular species was noticeably different from those of PC in developing SASK. Other noteworthy species were 16:1/16:1 and 18:1/18:2, which showed little change in proportion during SASK development (Figure 4B).

Utilizing PA from the Kennedy pathway, phosphatidylglycerol (PG) is synthesized. The main molecular species of PA included 22:0/18:1, 22:4/18:3, 22:2/18:2, 16:0/18:1 and 16:0/18:2 (Figure 4C and Supplementary Table S6). The species of 22:2/18:2 and 16:0/18:1 had the maximum values at 6 WAA, while 22:0/18:1, 16:0/18:2 and 22:4/18:3 decreased during SASK development (Figure 4C). Intriguingly, the VLCFAs were the main composition, which were significantly different from PE and PC. This, along with minor amounts of VLCFAs in PA, DAG and TAG, suggests that PG is not involved in TAG accumulation.

\section{Acyl fluxes through PC may determine TAG FA composition}

As described above, three mechanisms allow the flux of FA through PC for eventual TAG synthesis [9-11]. First, PC acyl editing (Figure 5, orange arrows). The fatty acid desaturase 2 (FAD2) [25] and FAD3 [26] convert PC-bound oleate (C18:1) to linoleate (C18:2) and then linolenate (C18:3), respectively. The qRT-PCR data indicated that the FAD2 gene displayed a bell-shaped pattern of expression during the oil accumulation period (between 4 and 8 WAA), while FAD 3 genes showed a low expression level (Figure 6A). This suggests, as noted for FA compositions in developing SASK (Table 1), that linoleic acid rather than linolenic acid is a major composition in SASK oil. Results also indicate that the PC acyl editing is necessary for SASK oil accumulation. 
Second, acyl chains on PC can also be incorporated into the $s n-1$ and $s n-2$ positions of DAG via two known enzymatic routes (Figure 5, blue arrows). Our qRTPCR results indicated the expression levels of PDCT and DAG-CPT both displayed a downtrend with SASK development (Figure 6A). Surprisingly, the main species of 18:3/18:3 DAG also showed a notable decrease during SASK development (Figure 3A). Considering the lack of 18:3/18:3 species in PA (Figure 3B), it seems plausible that PDCT and DAG-CPT can generate 18:3/18:3 DAG from PC.

Third, the final step in TAG synthesis can also be catalyzed by PDAT (Figure 5, purple arrows), using the $s n-2$ and $s n-1$ (at a quarter of the rate for the $s n-2$ position) acyl group from PC [27]. PDATs from Arabidopsis and other plants have been shown to have high activity with PC containing unsaturated FAs [28, 29]. With this knowledge, we sought to use the lipidomic data to evaluate the possible contribution of PDAT to TAG accumulation. If the oilseeds are in the period of high oil biosynthetic rate, the flow of FAs is mostly into oil accumulation, and is not unduly influenced by other lipid metabolism [24]. Thus, these lipidomic data from 6 WAA (high biosynthetic rate) were chosen for evaluating the PDAT role in TAG biosynthesis. Using the sn-2 and $s n-1$ (a quarter of rate) acyl group of PC as the acyl donor, we assumed the ratios of C18:1/ C18:2 and C18:2/ C18:3. Also, the rates of C18:1/ C18:2 and C18:2/ C18:3 from the sn-1, sn-2and sn-3 acyl group of TAG were evaluated. As expected, the rates of C18:1/ C18:2 and C18:2/ C18:3 from the sn-1and sn-2 of TAG had enormous differences with those from sn-2 of PC in both AS-80 and AS-84 (Figure 6B). These data suggest that PDAT does not function in sn-1and sn-2 acylation of TAG. Although the C18:1/ C18:2 rate showed a substantial difference between sn-3 of TAG and sn-2 of PC, it was worth noting that the C18:2/ C18:3 rate from $s n-3$ of TAG was close to this from sn-2 of PC in both AS-80 and AS-84 (Figure 6B). Our data implicate involvement of PDAT in sn-3 acylation of TAG, and strong substrate selectivity for PUFAs by PDAT enzyme.

In summary, the above-mentioned results imply the importance of PC-mediated "PUFA trafficking" for TAG biosynthesis, which may determine the FA composition of TAG in developing SASK.

\section{Discussion}

Plant oil is composed of long chain hydrocarbons, and thus can also replace petroleum in many applications, such as feedstock for chemical and biofuel. Owing to its important uses, high value and increased demand, oil biosynthesis in plant seed has been extensively studied. Numerous studies in plants have elucidated the biochemical pathways producing storage lipids, namely TAGs [7, 8, 19, 30]. In Arabidopsis, 120 enzymatic reactions and more than 600 genes were divided into 12 sections based on the types of lipids produced and their subcellular localization [31]. Although our recent studies in developing SASK have provided details on the very large number of genes involved in FA synthesis and TAG biosynthesis [12-14], some detailed metabolisms remain largely unknown such as the FA flux from the basic Kennedy pathway into and out of PC [32]. To extend and complement our knowledge about the regulation of lipid accumulation, we conducted the detailed lipidomic examination of important lipid classes during SASK development.

The plastid FA synthesis pathway is catalyzed by various enzymes responsible for producing chain lengths from 6 to 18 carbons [7]. Some 18:0-ACP are efficiently desaturated by a stearoyl-ACP desaturase (SAD) [32]. Based on our previous transcriptomic analysis of $P$. sibirica, SAD6 was identified as the potential enzyme for oleic acid formation [12]. In both AS-80 and AS-84, SAD6 gene showed a bell-shaped pattern of expression (up-regulated expression during 5-7 WAA), but it was sharply up-regulated in AS-80 compared with AS-84 (Figure 6A). This could explain why AS-80 had a higher content of oleic acid (Table 1). These data coupled with our previous transcriptome [12] and co-expression studies [13] clearly indicated that SAD6 expression is important for directing the acyl-ACP flux toward the oleoyl-ACP synthesis in developing SASK. Acyl groups esterified to PC are the site of extra-plastidic FA desaturation, namely PC acyl editing [33]. This process involves reacylation of lyso-PC with an acyl-CoA from the acyl-CoA pool, and deacylation of PC which generates lyso-PC and releases the FA or acyl-CoA to the acyl-CoA pool (Figure 5) [31]. In endoplasmic reticulum, FAD2 [25] and FAD3 [26] can convert PC-oleate (C18:1) to PC-linoleate (C18:2) and then PC-linolenate (C18:3), respectively. The qRT-PCR analysis showed up-regulated expression for FAD2 and down-regulated expression for FAD3 during 5-7 WAA (Figure 6A), in agreement with our previous results [12]. Also, the temporal expression of FAD2 closely matched the cumulative rate of linoleic acid in both AS-80 and AS-84 (Table 1), corroborating the role of FAD2 in PC-mediated biosynthesis of linoleic acid. The PC acyl editing exchanges the FA on PC with the acyl-CoA pool (Figure 5), resulting in increasing PUFAs in acyl-CoA pool. Previous studies in developing soybean embryos revealed that about $60 \%$ of nascent acyl chains are incorporated directly into the $s n-2$ position of PC through acyl editing mechanism rather than through the sequential acylation converting to TAG [34].

The de novo assembly of TAG is initiated with two acylations of G3P by glycerol-3-phosphate acyltransferase (GPAT) and lysophosphatidic acid acyltransferase (LPAAT) [35]. Subsequently, phosphatidic acid (PA) phosphatase functions in the removal of the phosphate group from PA to form DAG [35]. In AS-80 and AS-84, despite PA is directly connected with DAG in Kennedy pathway, the pattern of molecular species in PA was significantly distinct from that of DAG (Figure 3), which was consistent with the lipidomics results in developing seeds from oilseed rape [24]. This may be caused by the complex outflux of PA. Indeed, PA also can be used for PG biosynthesis (Figure 5) [31]. In developing SASK, the 16:0/18:1 and 16:0/18:2 as the main molecular species of PA could be also found in PG (Figure 3B and 3C). Interestingly, 18:3/18:3 was the main molecular species in DAG, but PA lacked it. This difference may reflect the very significant flux of acyl chains between DAG and PC. The acyl chains on PC can also be reversibly incorporated into the sn-1 and sn-2 positions of DAG by PDCT [36] and DAG-CPT [37] (Figure 5). As mentioned above, FAD3 catalyzes the biosynthesis of linolenate (C18:3). During SASK development, the expression patterns of FAD3, PDCT and DAG-CPT showed a higher level at early development of AS-80 and AS-84 (Figure 6A), which was exactly similar to the cumulative pattern of 18:3/18:3 DAG (Figure 3A). Therefore, it is reasonable to presume that first PA-derived DAG is converted to PC, and then after PC acyl editing these unsaturated acyl chains from PC are transferred to generate newly synthesized DAG (Figure 5). In vivo metabolic flux analysis of soybeans strongly suggests that PA-derived DAG and PC-derived DAG are distinct pools that do not intermix [34]. The mutation of PDCT in Arabidopsis reduces 18:2 and 18:3 accumulation by $40 \%$ in seed [36]. These expression data of FAD3, PDCT and DAG-CPT also could explain the late decrease of 18:3/18:3 DAG in developing SASK. Taken together, our data suggest that PC acyl editing is important for the flux of PUFAs into PC-derived DAG.

Using DAG from the Kennedy pathway, PE is formed by diacylglycerol ethanolaminephosphotransferase (DAG-EPT) [38]. Previous studies in soybean suggested that DAG-CPT and DAG-EPT have been demonstrated to have distinct characteristics, but an isolated gene codes for both activities [39]. PE can

Page 4/15 
also be converted to PC by methylation [40]. Lipidomics data showed that 18:1/18:1 and 18:1/18:2 were the main molecular species in PC (Figure 4A), whereas 16:0/18:3, 18:1/18:1 and 16:0/18:1 were the main species in PE (Figure 4B). The distribution of molecular species in PC and PE is clearly different. Additionally, the contents of 18:1/18:1 and 18:1/18:2 PC in AS-80 was higher than that in AS-84 at 6 WAA, while the content of main PE species had no substantial difference between AS-80 and AS-84 (Figure 4B). These data imply PC rather than PE as a central intermediate destined to FA desaturation and TAG accumulation in developing SASK, in keeping with the previous conclusion [41].

As remarked before, although there is a closely metabolic connection among PA, PG, PC and PE (Figure 5) [31, 36-38], all of them had notable differences in distribution of molecular species and the changes to their molecular species in developing SASK. For example, the acyl chains of PG were mainly composed of VLCFAs (Figure 4C), while only minor amounts of PA, PC and PE contained VLCFAs (Figure 3B, 4A and 4B). The 18:1/18:1 species of PA and PE both had a highest level at 6 WAA (Figure 3B and 4C), but the maximum values of PC were at 8 WAA (Figure 4A). Similarly, various molecular species from PA, PG, PC and PE showed differently temporal changes. These subtle distinctions in metabolism for phosphoglycerides may reflect their different functions in plant cells [42].

In plants, at least three mechanisms differing in their acyl donor sources have been identified to be involved in the final acylation reaction [31]. In its simplest form, DGAT catalyze the last acylation of Kennedy pathway using a acyl-CoA molecule [43]. Second, DAG can be acylated by PDAT using PC as the acyl donor [44]. Third, studies in Carthamus tinctorius seeds implied the existence of diacylglycerol:diacylglycerol transacylase activity that acylate DAG to form TAG using DAG as the acyl donor [45], but the enzymes catalyzing such activity has yet to be identified. Although there are multiple TAG biosynthetic pathways,

DAG is the direct substrate for TAG formation. In sharp contrast to the compositions of acyl chains for phosphoglycerides, the acyl chains for DAG was lack of palmitoyl (Figure 3A). This is to be expected from lipidomic data of TAG, minor amounts of TAG species containing palmitoyl (Figure 2). However, the most striking difference with regards to the major species of TAG and DAG concerned 18:3/18:3. At the mid-point (6 WAA) of oil accumulation, 18:3/18:3 represented one of the highest counts of all molecular species of DAG in AS-80 and AS-84 (Figure 3A), whereas TAG lack the molecular species of 18:3/18:3/* (Figure 2). These data suggest the substrate selectivity of DGAT and PDAT, and/or the presence of separate pools of DAG, as discussed above in metabolic flux analysis of soybeans [34].

DGAT and PDAT are the key enzymes involved in the final step of TAG synthesis in plants. However, their relative contribution to TAG accumulation has not been determined in most oilseed plants [8]. Mutant studies in Arabidopsis have shown that the PDAT1 mutant have no effect on TAG accumulation [16], while the DGAT1 mutant only reduces oil content by $20-30 \%$ [46]. However, a double knockout of PDAT1 and DGAT1 is lethal, and RNAi suppression of PDAT1 gene in DGAT1 mutant results in severe defects in pollen and seed development, including $80 \%$ reduction of oil content [17]. From these results, the overlapping functions of DGAT and PDAT in oil accumulation can be concluded. The absence of DGAT is partially compensated by PDAT, but DGAT fully compensate for a lack of PDAT. Based on our previous transcriptomic analysis, it was identified that DGAT1 and PDAT2 may play a major role in TAG synthesis in developing SASK [12]. Indeed, the genes encoding DGAT1 and PDAT2 display a bell-shaped pattern of expression with high expression during the rapid period of oil accumulation (Figure 6A), suggesting the cooperative role of DGAT1 and PDAT2 in TAG synthesis. Although our preceding studies showed that the transcription level of PDAT2 was much lower than that of DGAT1 [12], the expression level cannot be used to elucidate the relative contribution of the two enzymes in TAG accumulation. As was reported in a recent review, the relative importance of DGAT versus PDAT is a major uncertainty in plant lipid metabolism [32]. In this study, using the lipidomics data, we tried to evaluate the role of PDAT2 in TAG synthesis. Under this assumption that unsaturated FAs at the $s n-2$ and $s n-1$ (at a quarter of the rate for the sn-2 position) position of PC can be utilized by PDAT [44], the C18:1/ C18:2 and C18:2/ C18:3 rates from $s n-2$ of PC and $s n-1, s n-2$ and $s n-3$ of TAG were calculated. One conclusion can be proposed that PDAT2 may have substrate specificity, resulting in that PDAT2 preferentially utilized PUFAs from PC. In sunflower and safflower seeds, the specialisation towards 18:2 in sn-2 position of PC displayed by PDAT, and in case of safflower PDAT had 3.5 times higher activity for 18:2 than 18:1 [27]. To a certain extent, these results indicated that PDAT may play a critical role in incorporation of PUFAs into sn-3 of TAG.

\section{Conclusions}

In this study, two germplasms of $P$. sibirica (AS-80 and AS-84) with significant differences in oil content were used for plant material. According to the temporal patterns of oil and FAs in developing SASK, the optimal samples from 4, 6 and 8 WAA that respectively represent early, mid and late phases of oil accumulation were identified for lipidomic analysis. To our knowledge, this is the first report on lipidomic analysis of major lipids involved in TAG formation during seed development in woody oilseed plants. The lipidomic results showed quite distinct molecular species distribution in PA, DAG, PC, PE, PG and TAG, reflecting the complexity of lipid flux metabolism during oil accumulation. Moreover, the lipidomic data coupled with qRT-PCR analysis indicated that PC is a central intermediate in the flux of PUFAs or DAG, or both substrates destined to TAG synthesis in developing SASK. All of these findings will be conducive to understand the underlying mechanisms how the storage lipid is formed in developing SASK, and may also provide some reference for researching the wood oilseed plants.

\section{Methods Plant material}

Based on the collection and identification of $P$. sibirica germplasm resources, two germplasms (AS-80 and AS-84) with significant differences in oil content were used plant material in this study. AS-80 and AS-84 locating in Changping District of Beijing (geographical coordinates approximately $116^{\circ} 23^{\prime} \mathrm{E}, 40^{\circ} 22^{\prime}$ N) were selected and marked in April 2018. Fruits were collected at 3, 4, 5, 6, 7, 8 and 9 weeks after anthesis (WAA). After removing the pericarps, the seeds were immediately frozen in liquid nitrogen and stored at $-80^{\circ} \mathrm{C}$ until use. 


\section{Oil extraction and component analysis}

First, the SASKs were dried and crushed. The oils were extracted by n-hexane double extraction at $25^{\circ} \mathrm{C}$ using a rotary evaporator (LABORTA 4000 -efcient, Heidolph, Germany). Oil content was determined by comparing the weight of dry kernel before and after extraction. The extracted oil was methylated following a previous method [5]. The obtained fatty acid methyl esters (FAME) were analyzed by GC-MS, using the Agilent 6890 equipped with a fame ionization detector (California, USA). The following chromatographic conditions were applied: inlet temperature $250{ }^{\circ} \mathrm{C}$, detector temperature $280{ }^{\circ} \mathrm{C}$ and injection volume $1 \mu \mathrm{L}$. The carrier gas was high-purity hydrogen. Peak integration was performed by applying HP3398A software. Qualitative and quantitative analyses were determined using a FAME mix as standard sample (ANPEL, Shanghai, China). All the determination was performed in triplicate, and the data reported as mean \pm standard deviation.

\section{Lipid extraction}

The SASKs from 4, 6 and 8 WAA, which respectively represent early, mid and late phases of oil accumulation, were selected as the optimal period for lipidomic analysis. Each biological repeat formed from at least three separate SASKs. $20 \mathrm{mg}$ of SASK sample was weighted to an EP tube, and homogenized with 400 $\mu \mathrm{L}$ water. $200 \mu \mathrm{L}$ of the homogenate was transferred to a fresh EP tube, and add water to $400 \mu \mathrm{L}$. Then $960 \mu \mathrm{L}$ extract solution (MTBE:methanol = 5:1)

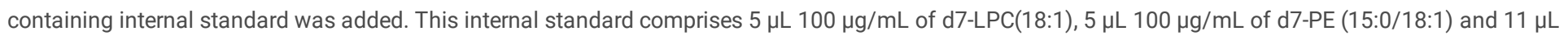
$100 \mu \mathrm{g} / \mathrm{mL}$ of d7-TAG(15:0/18:1/15:0). All standards were purchased from Avanti Polar Lipids (Alabaster, USA). After $30 \mathrm{~s}$ vortex, the samples were sonicated for $10 \mathrm{~min}$ in ice-water bath, followed by centrifuged at $4{ }^{\circ} \mathrm{C} 3000 \mathrm{rpm}$ for $15 \mathrm{~min} .500 \mu \mathrm{L}$ of supernatant was transferred to a fresh tube. The residual was added $500 \mu \mathrm{L}$ MTBE again, followed with vortex, sonication and centrifugation, and another $500 \mu \mathrm{L}$ of supernatant was taken out. This step was repeated once. The three supernatants were combined and dried in a vacuum concentrator at $37^{\circ} \mathrm{C}$. The dried samples were reconstituted in $200 \mu \mathrm{L}$ of $50 \%$ methanol in dichloromethane by sonication on ice for $10 \mathrm{~min}$. After centrifugation at $4{ }^{\circ} \mathrm{C} 1300 \mathrm{rpm}$ for $15 \mathrm{~min}, 75 \mu \mathrm{L}$ of supernatant was transferred to a fresh glass vial for LC-MS/MS analysis. The quality control sample was prepared pooling $20 \mu \mathrm{L}$ of each sample together.

\section{LC-MS/MS analysis}

The target compounds were separated by 1290 Infinity series UHPLC System (Agilent Technologies), equipped with a Kinetex C18 column (2.1 * 100 mm, 1.7 $\mu \mathrm{m}$, Phenomen). The mobile phase A was composed of $40 \%$ water, $60 \%$ acetonitrile, and $10 \mathrm{mmol} / \mathrm{L}$ ammonium formate. The mobile phase B consisted of $10 \%$ acetonitrile and $90 \%$ isopropanol, which was added with $50 \mathrm{~mL} 10 \mathrm{mmol} / \mathrm{L}$ ammonium formate for every $1000 \mathrm{~mL}$ mixed solvent. The analysis was carried with elution gradient as follows: 0 12.0 min, 40\% 100\% B; 12.0 13.5 min, 100\% B; 13.5 13.7 min, 100\% 40\% B; 13.7 18.0 min, 40\% B. The column temperature was $45^{\circ} \mathrm{C}$. The auto-sampler temperature was $4{ }^{\circ} \mathrm{C}$, and the injection volume was $0.5 \mu \mathrm{L}$ (pos) or $2 \mu \mathrm{L}$ (neg), respectively.

Triple TOF 6600 (AB Sciex, USA) was used for its ability to acquire MS/MS spectra on an information-dependent basis during an LC/MS experiment. The acquisition software (Analyst TF 1.7, AB Sciex) continuously evaluates the full scan survey MS data as it collects and triggers the acquisition of MS/MS spectra depending on preselected criteria. In each cycle, the most intensive 12 precursor ions with intensity above 100 were chosen for MS/MS at collision energy of $45 \mathrm{eV}$ (12 MS/MS events with accumulation time of $50 \mathrm{msec}$ each). ESI source conditions were set as following: Gas 1 as 60 psi, Gas 2 as 60 psi, Curtain Gas as $30 \mathrm{psi}$, Source Temperature as $600^{\circ} \mathrm{C}$, Declustering potential as $100 \mathrm{~V}$, lon Spray Voltage Floating as $5000 \mathrm{~V}$ (pos) or -4500 V (neg).

\section{Lipidomic data processing}

The data were analyzed by Biomarker (Beijing, China) as follows. The raw data files (.wiff format) were converted to files in $\mathrm{mzXML}$ format using the 'msconvert' program from ProteoWizard (version 3.0.6150). Then, the mzxML files were loaded into LipidAnalyzer for data processing. Peak detection was first applied to the MS1 data. The CentWave algorithm in XCMS was used for peak detection, With the MS/MS spectrum, lipid identification was achieved through a spectral match using an in-house MS/MS spectral library (Biomarker, Beijing, China). The relative quantitation of lipids can be achieved using the peak area, SIL-IS and RF information.

\section{qRT-PCR analysis}

Based on our previous transcriptomic data of $P$. sibirica [12], the amplification primers were designed using PrimerQuest (www.idtdna.com/PrimerQuest/Home/Index) sofware with melting temperatures at $62^{\circ} \mathrm{C}$. The qRT-PCR was performed using the SYBR Premix Ex Taq Kit (TaKaRa, Liaoning, China) according to the manufacturer's protocol. According to our previous studies, cyclophilin and ubiquitin-conjugating enzyme were used as reference genes in SASK [47]. The qRT-PCR primer sequences were shown in Supplementary Table S7. The relative expression levels were calculated

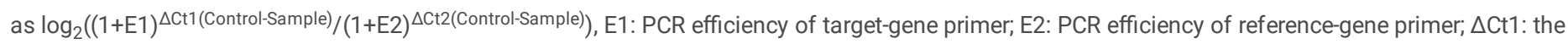
difference of $\mathrm{Ct}$ value between control and sample in experimental group; $\Delta \mathrm{Ct} 2$ : the difference of $\mathrm{Ct}$ value between control and sample in reference group. The PCR efficiency ( $E$ ) was estimated by the equation of $E=10^{\text {slope }}$ from the data obtained from each individual amplification plot [48]. The statistical analysis of expressed data was performed using SPSS software.

\section{Abbreviations}


diacylglycerol, DAG; phospholipid:DAG acyltransferase, PDAT; diacylglycerol acyltransferase, DGAT; diacylglycerol cholinephosphotransferase, DAG-CPT; diacylglycerol ethanolaminephosphotransferase, DAG-EPT; fatty acid, FA; fatty acid methyl esters, FAME; fatty acid desaturase, FAD; glycerol-3-phosphate, G3P; glycerol-3-phosphate acyltransferase, GPAT; liquid chromatography-tandem mass spectrometry, LC-MS/MS; lysophosphatidic acid acyltransferase, LPAAT; phosphatidic acid, PA; phosphatidylcholine, PC; phosphatidylcholine: diacylglycerol cholinephosphotransferase, PDCT; phosphatidyl-ethanolamine, PE; phosphatidylglycerol PG; polyunsaturated fatty acids, PUFAs; quantitative reverse transcription PCR, qRT-PCR; Siberian apricot seed kernel, SASK; stearoyl-ACP desaturase, SAD; triacylglycerol, TAG; very long-chain fatty acids, VLCFAs; weeks after anthesis, WAA.

\section{Declarations}

\section{Ethics approval and consent to participate}

Not applicable.

\section{Consent for publication}

Not applicable

\section{Availability of data and materials}

Not applicable.

\section{Competing interests}

The authors declare that they have no competing interests.

\section{Funding}

The author acknowledges the financial support of the Central Public-Interest Scientific Institution Basal Research Fund (CAFYBB2017ZA004-4), the National Natural Science Foundation of China (31700586 and 41401069) and Hainan University research funded projects (KYQD(ZR)1701).

\section{Authors' contributions}

JN and LW conceived and designed the experiments. YM and KH wrote the manuscript and collected material. HY, NZ, SL and YL performed the lipidomics analysis. NW and CZ conducted the qRT-PCR analysis.

\section{Acknowledgements}

Not applicable.

\section{Supplementary Data}

Supplementary Table S1. Changes in molecular species of TAG during SASK development.

Supplementary Table S2. Changes in molecular species of DAG during SASK development.

Supplementary Table S3. Changes in molecular species of PA during SASK development.

Supplementary Table S4. Changes in molecular species of PC during SASK development.

Supplementary Table S5. Changes in molecular species of PE during SASK development.

Supplementary Table S6. Changes in molecular species of PG during SASK development.

Supplementary Table S7 The primers for qRT-PCR analysis.

\section{References}

1.Wang L: Resource investigation and distribution pattern of three Armeniaca species. Forest Resour Manage 2011, 5:65-70.

2.Zhang J: Chinese Fruit Species, vol. Apricot Volume. Beijing: China Forestry Publishing House; 2003.

3.Wang L: Evaluation of Siberian Apricot (Prunus sibirica L.) germplasm variability for biodiesel properties. J Am Oil Chem Soc 2012, 89(9):1743-1747. 
4.Guo F, Fang Z, Tian XF, Long YD, Jiang LQ: One-step production of biodiesel from Jatropha oil with high-acid value in ionic liquids. Bioresour Technol 2011, 102:6469-6472.

5.Wang LB, Hai-Yan YU, Xiao-Hui HE, Liu RY: Influence of fatty acid composition of woody biodiesel plants on the fuel properties. J Fuel Chem Tech 2012, 40(4):397-404.

6.Ruan YL: Sucrose metabolism: gateway to diverse carbon use and sugar signaling. Annu Rev Plant Biol 2014, 65(1):33.

7.Baud S, Lepiniec L: Regulation of de novo fatty acid synthesis in maturing oilseeds of Arabidopsis. Plant Physiol Bioch 2009, 47(6):448-455.

8.Bates PD, Stymne S, Ohlrogge J, Browse J, Farmer E: Biochemical pathways in seed oil synthesis. Curr Opin in Plant Bio 2013, $16(3): 358$.

9.Bates PD, Browse J: The significance of different diacylgycerol synthesis pathways on plant oil composition and bioengineering. Front Plant Sci 2012, $3(4): 147$.

10.Bates PD, Abdelhak F, Snapp AR, Carlsson AS, John B, Chaofu L: Acyl editing and headgroup exchange are the major mechanisms that direct polyunsaturated fatty acid flux into triacylglycerols. Plant Physiol 2012, 160(3):1530-1539.

11.Ulf S, Carlsson AS, Marit L, Anders D, Bangquan H, Walentyna B, Antoni B, Sten S: Cloning and functional characterization of a phospholipid:diacylglycerol acyltransferase from Arabidopsis. Plant Physiol 2004, 135(3):1324-1335.

12.Niu J, An J, Wang L, Fang C, Denglong H, Fu C, Qiu L, Yu H, Zhao H, Hou X: Transcriptomic analysis revealed the mechanism of oil dynamic accumulation during developing Siberian apricot (Prunus sibirica L.) seed kernels for the development of woody biodiesel. Biotechnol Biofuels 2015, 8(1):29.

13.Deng S, Mai Y, Shui L, Niu J: WRINKLED1 transcription factor orchestrates the regulation of carbon partitioning for C18_1 (oleic acid) accumulation in Siberian apricot kernel. SCI REP-UK 2019, 9.2693.

14.Niu J, Wang J, An J, Liu L, Lin Z, Wang R, Wang L, Ma C, Shi L, Lin S: Integrated mRNA and miRNA transcriptome reveal a cross-talk between developing response and hormone signaling for the seed kernels of Siberian apricot. SCI REP-UK 2016, 6:35675.

15.Zou J,., Wei Y,., Jako C,., Kumar A,., Selvaraj G,.., Taylor DC: The Arabidopsis thaliana TAG1 mutant has a mutation in a diacylglycerol acyltransferase gene. Plant J 2010, 19(6):645-653.

16.Mhaske V, Beldjilali K, Ohlrogge J, Pollard M: Isolation and characterization of an Arabidopsis thaliana knockout line for phospholipid: diacylglycerol transacylase gene (At5g13640). Plant Physiol Biochem 2005, 43(4):413-417.

17.Meng Z, Jilian F, Taylor DC, Ohlrogge JB: DGAT1 and PDAT1 acyltransferases have overlapping functions in Arabidopsis triacylglycerol biosynthesis and are essential for normal pollen and seed development. Plant Cell 2009, 21(12):3885-3901.

18.Mingguo T, Guschina IA, Paul OH, Slabas AR, Quant PA, Tony F, Harwood JL: Metabolic control analysis of developing oilseed rape (Brassica napus cV Westar) embryos shows that lipid assembly exerts significant control over oil accumulation. New Phytol 2012, 196(2):414-426.

19.Troncoso-Ponce MA, Kilaru A, Cao X, Durrett TP, Fan J, Jensen JK, Thrower NA, Pauly M, Wilkerson C, Ohlrogge JB: Comparative deep transcriptional profiling of four developing oilseeds. Plant J 2011, 68(6):1014-1027.

20.Parviz M, Atiyeh M, Maryam K, Marcello I: Lipidomics Unravels the Role of Leaf Lipids in Thyme Plant Response to Drought Stress. Int J Mol Sci 2017, 18(10):2067-.

21.Liu MY, Burgos A, Ma L, Zhang Q, Tang D, Ruan J: Lipidomics analysis unravels the effect of nitrogen fertilization on lipid metabolism in tea plant (Camellia sinensis L.). Bmc Plant Biol 2017, 17(1):165.

22.Horn PJ, Chapman KD: Lipidomics in tissues, cells and subcellular compartments. P J Cell Mol Biol 2012, 70(1):69-80.

23.Okazaki Y, Kamide Y, Hirai MY, Saito K: Plant lipidomics based on hydrophilic interaction chromatography coupled to ion trap time-of-flight mass spectrometry. Metabolomics 2013, 9:121-131.

24.Woodfield HK, Cazenave-Gassiot A, Haslam RP, Guschina IA, Wenk MR, Harwood JL: Using lipidomics to reveal details of lipid accumulation in developing seeds from oilseed rape (Brassica napus L.). BBA-Mol Cell Biol L 2018, 1863:339-348.

25. Okuley J, Lightner J, Feldmann K, Yadav N, Lark E, Browse J: Arabidopsis FAD2 gene encodes the enzyme that is essential for polyunsaturated lipid synthesis. Plant Cell 1994, 6:147-158.

26.Browse J,., Mcconn M,., James D,., Miquel M,. Mutants of Arabidopsis deficient in the synthesis of alpha-linolenate. Biochemical and genetic characterization of the endoplasmic reticulum linoleoyl desaturase. J Biol Chem 1993, 268(22):16345-16351.

27.Bana W, Garcia AS, Bana A, Stymne S: Activities of acyl-CoA:diacylglycerol acyltransferase (DGAT) and phospholipid:diacylglycerol acyltransferase (PDAT) in microsomal preparations of developing sunflower and safflower seeds. Planta 2013, 237(6):1627-1636. 
28.Van Erp H, Bates PD, Burgal J, Shockey J, Browse J: Castor Phospholipid:Diacylglycerol Acyltransferase Facilitates Efficient Metabolism of Hydroxy Fatty Acids in Transgenic Arabidopsis. Plant Physiol, 155(2):683-693.

29.Kim HU, Lee K-R, Go YS, Jung JH, Suh M-C, Kim JB: Endoplasmic Reticulum-Located PDAT1-2 from Castor Bean Enhances Hydroxy Fatty Acid Accumulation in Transgenic Plants. Plant Cell Physiol 2011, 52(6):983-993.

30.Bourgis F, Kilaru A, Cao X, Ngando-Ebongue G-F, Drira N, Ohlrogge JB, Arondel V: Comparative transcriptome and metabolite analysis of oil palm and date palm mesocarp that differ dramatically in carbon partitioning. Proc Natl Acad Sci USA 2011, 108(30):12527-12532.

31.Li-Beisson Y, Shorrosh B, Beisson F, Andersson MX, Ohlrogge J: Acyl-Lipid Metabolism. The Arabidopsis Book 2013, 11(e0161).

32.Bates, D. P: Understanding the control of acyl flux through the lipid metabolic network of plant oil biosynthesis. Biochim Biophys Acta 2016, 1861:12141225.

33.Speerling P, Heinz E: Isomeric sn-1-octadecenyl and sn-2-octadecenyl analogues of lysophosphatidylcholine as substrates for acylation and desaturation by plant microsomal membranes. Eur J Biochem 2005, 213(3):965-971.

34.Bates PD, Durrett TP, Ohlrogge JB, Pollard M: Analysis of Acyl Fluxes through Multiple Pathways of Triacylglycerol Synthesis in Developing Soybean Embryos. Plant Physiol 2009, 150(1):55-72.

35.Barron EJ, Stumpf PK: Fat metabolism in higher plants XIX. The biosynthesis of triglycerides by avocado-mesocarp enzymes. Biochim Biophys Acta 1962, 60(2):329-337.

36.Lu C, Xin Z, Ren Z, Miquel M, Browse J: An enzyme regulating triacylglycerol composition is encoded by the ROD1 gene of Arabidopsis. Proc Natl Acad Sci USA 2009, 106(44):18837-18842.

37.Slack CR, Campbell LC, Browse JA, Roughan PG: Some evidence for the reversibility of cholinephosphotransferase-catalyzed reaction in developing linseed cotyledons in vivo. Biochim Biophys Acta 1983, 754(1):10-20.

38.Kennedy EP: Biosynthesis of complex lipids. Fed Proc 1961, 20:934-940.

39.Harwood JL: Synthesis of molecular species of phosphatidylcholine and phosphatidylethanolamine by germinating soya bean. Phytochemistry 1976, 15(10):1459-1463.

40.Williams M, Harwood JL: Alternative pathways for phosphatidylcholine synthesis in olive (Olea europaea L.) callus cultures. Biochem J 1994, 304 463468.

41.Chen G, Woodfield HK, Pan X, Harwood JL, Weselake RJ: Acyl-Trafficking During Plant Oil Accumulation. Lipids 2015, 50(11):1057-1068.

42.Viola-Magni MP, Gahan PB, Pacy J: Phospholipids in plant and animal cells. Cell Biochem Funct 1985, 3(1):71-78.

43.Liu Q, Siloto RMP, Lehner R, Stone SJ, Weselake RJ: Acyl-CoA:diacylglycerol acy/transferase: Molecular biology, biochemistry and biotechnology. Prog Lipid Res 2012, 51(4):350-377.

44.Dahlqvist A, Stahl U, Lenman M, Banas A, Lee M, Sandager L, Ronne H, Stymne S: Phospholipid:diacylglycerol acyltransferase: An enzyme that catalyzes the acyl-CoA-independent formation of triacylglycerol in yeast and plants. Proc Natl Acad Sci USA 2000, 97(12):6487-6492.

45.Stobart K, Mancha M, Lenman M, Dahlqvist A, Stymne S: Triacylglycerols are synthesised and utilized by transacylation reactions in microsomal preparations of developing safflower (Carthamus tinctorius L.) seeds. Planta 1997, 203(1):58-66.

46.Zou J, Wei Y, Jako C, Kumar A, Taylor DC: The Arabidopsis thaliana TAG1 mutant has a mutation in a diacylglycerol acyltransferase gene. Plant J 1999 , 19(6):645-653.

47.Niu J, Zhu B, Cai J, Li P, Wang L, Dai H, Qiu L, Yu H, Ha D, Zhao H: Selection of reference genes for gene expression studies in Siberian Apricot (Prunus sibirica L.) Germplasm using quantitative real-time PCR. Plos One 2014, 9(8):e103900.

48.Ramakers C, Ruijter JM, Deprez RH, Moorman AF: Assumption-free analysis of quantitative real-time polymerase chain reaction (PCR) data. Neuroscience Lett 2003, 339(1):62-66.

\section{Table}

\section{Table 1}

Changes in FA compositions in developing SASK 


\begin{tabular}{|c|c|c|c|c|c|c|c|c|c|c|c|}
\hline & AS-80 & & & & & & & AS-84 & & & \\
\hline & $3 W A A$ & 4WAA & 5WAA & 6WAA & 7WAA & 8WAA & 9WAA & 3WAA & 4WAA & 5WAA & 61 \\
\hline $\begin{array}{l}\text { Palmitic } \\
\text { acid } \\
\text { (C16:0) }\end{array}$ & $0.31 \pm 0.02$ & $0.38 \pm 0.03$ & $0.98 \pm 0.09$ & $1.24 \pm 0.12$ & $1.54 \pm 0.07$ & $1.77 \pm 0.16$ & $1.65 \pm 0.04$ & $0.24 \pm 0.02$ & $0.28 \pm 0.03$ & $0.58 \pm 0.04$ & 1. \\
\hline $\begin{array}{l}\text { Palmitoleic } \\
\text { acid } \\
\text { (C16:1) }\end{array}$ & $0.02 \pm 0.00$ & $0.06 \pm 0.01$ & $0.16 \pm 0.01$ & $0.20 \pm 0.06$ & $0.23 \pm 0.02$ & $0.26 \pm 0.02$ & $0.25 \pm 0.01$ & $0.01 \pm 0.00$ & $0.07 \pm 0.01$ & $0.07 \pm 0.00$ & 0. \\
\hline $\begin{array}{l}\text { Stearic } \\
\text { acid } \\
\text { (C18:0) }\end{array}$ & $0.05 \pm 0.01$ & $0.05 \pm 0.00$ & $0.15 \pm 0.03$ & $0.26 \pm 0.05$ & $0.43 \pm 0.04$ & $0.47 \pm 0.03$ & $0.46 \pm 0.03$ & $0.06 \pm 0.00$ & $0.09 \pm 0.01$ & $0.09 \pm 0.01$ & 0. \\
\hline $\begin{array}{l}\text { Oleic acid } \\
\text { (C18:1) }\end{array}$ & $0.14 \pm 0.02$ & $1.48 \pm 0.16$ & $6.31 \pm 036$ & $14.96 \pm 1.49$ & $26.01 \pm 1.49$ & $32.01 \pm 1.63$ & $32.69 \pm 3.04$ & $0.22 \pm 0.01$ & $1.36 \pm 0.12$ & $5.80 \pm 0.33$ & $1:$ \\
\hline $\begin{array}{l}\text { Linoleic } \\
\text { acid } \\
\text { (C18:2) }\end{array}$ & $0.56 \pm 0.04$ & $2.41 \pm 0.49$ & $7.28 \pm 0.29$ & $10.91 \pm 0.84$ & $15.80 \pm 1.12$ & $16.82 \pm 1.43$ & $15.48 \pm 2.74$ & $0.44 \pm 0.03$ & $3.00 \pm 0.22$ & $6.16 \pm 0.48$ & 8. \\
\hline $\begin{array}{l}\text { Linolenic } \\
\text { acid } \\
\text { (C18:3) }\end{array}$ & $0.07 \pm 0.00$ & $0.36 \pm 0.04$ & $0.46 \pm 0.04$ & $0.85 \pm 0.08$ & $1.45 \pm 0.12$ & $2.05 \pm 0.17$ & $2.04 \pm 0.09$ & $0.06 \pm 0.00$ & $0.37 \pm 0.02$ & $0.43 \pm 0.03$ & 0. \\
\hline $\begin{array}{l}\text { Arachidic } \\
\text { acid } \\
(\mathrm{C} 20: 0)\end{array}$ & - & $0.03 \pm 0.00$ & $0.03 \pm 0.00$ & $0.03 \pm 0.00$ & $0.04 \pm 0.00$ & $0.05 \pm 0.00$ & $0.04 \pm 0.00$ & - & $0.02 \pm 0.00$ & $0.02 \pm 0.00$ & 0. \\
\hline $\begin{array}{l}\text { Eicosenoic } \\
\text { acid } \\
(\mathrm{C} 20: 1)\end{array}$ & - & - & - & $0.03 \pm 0.00$ & $0.04 \pm 0.01$ & $0.05 \pm 0.00$ & $0.06 \pm 0.01$ & - & - & - & 0. \\
\hline
\end{tabular}

\section{Figures}

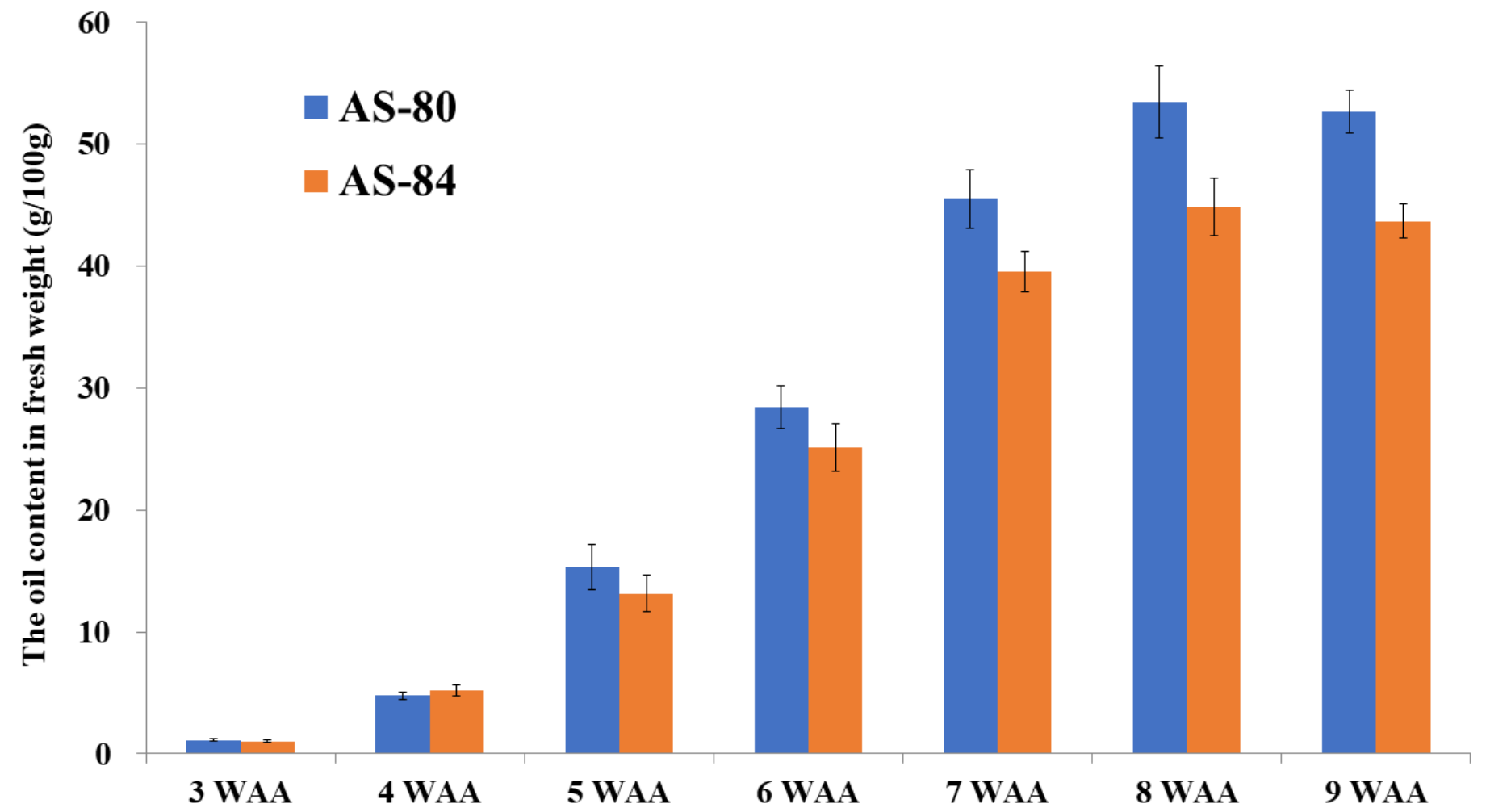

Figure 1

Variation of oil content in developing SASK. 


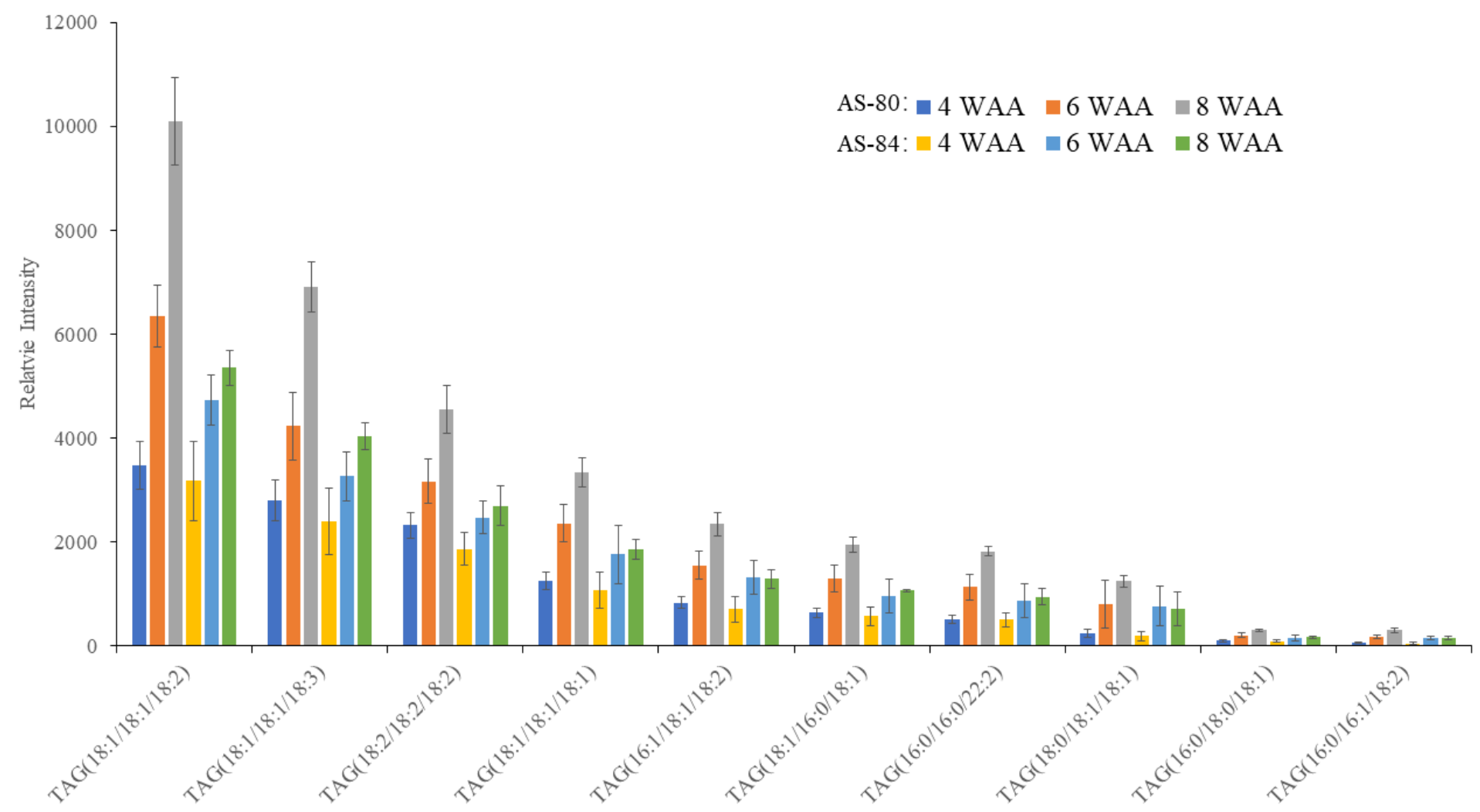

Figure 2

Relative content of the major molecular species of TAG during oil accumulation. The further information on minor species listed in Supplementary Table S2. 

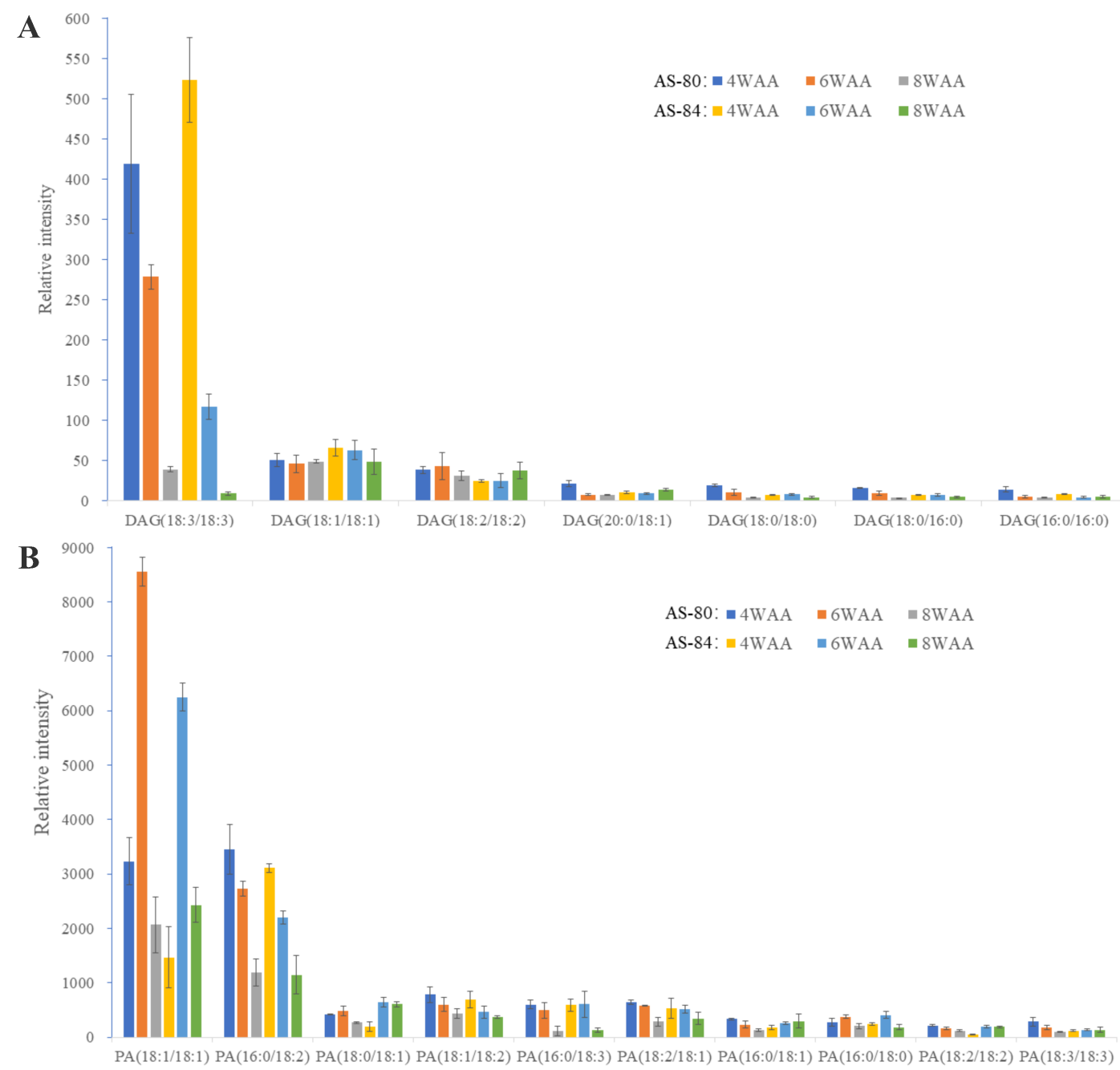

\section{Figure 3}

Relative content of the major molecular species of DAG (A) and PA (B) during oil accumulation. The further information on minor species listed in Supplementary Table S3 and S4, respectively. 


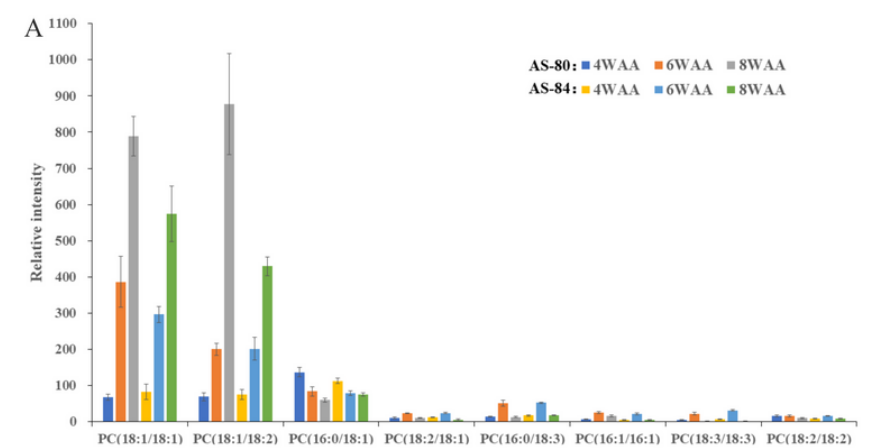

B
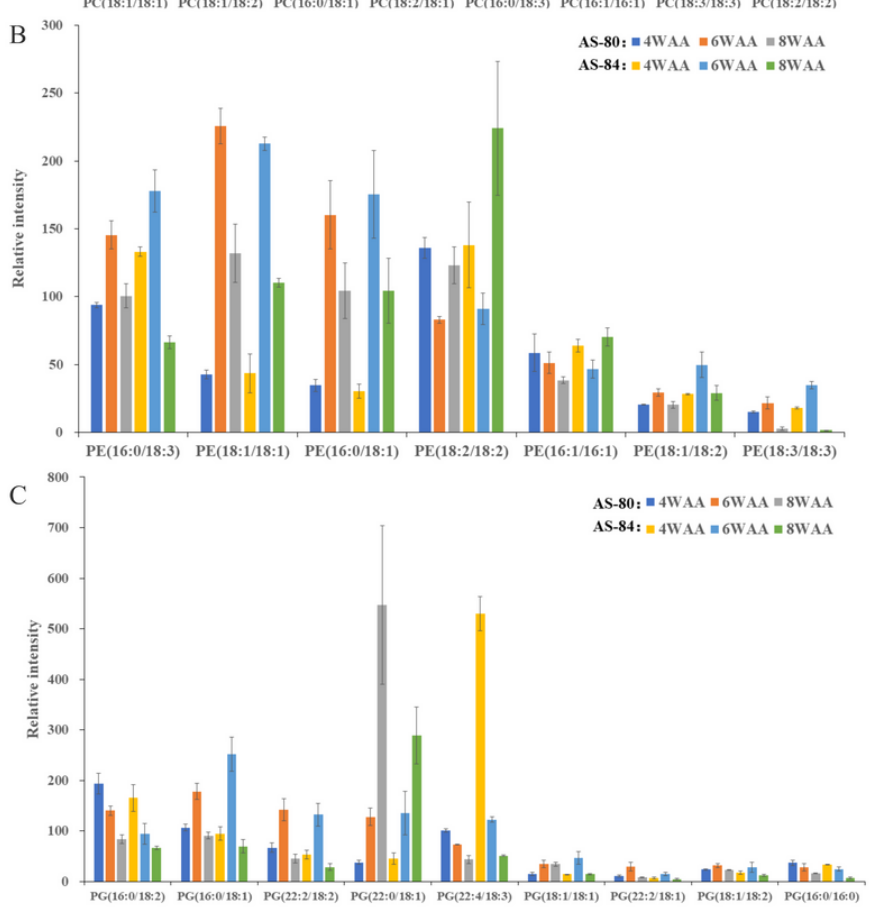

Figure 4

Relative content of the major molecular species of PC (A), PE (B) and PG (C) during oil accumulation. The further information on minor species listed in Supplementary Table S5, S6 and S7, respectively. 


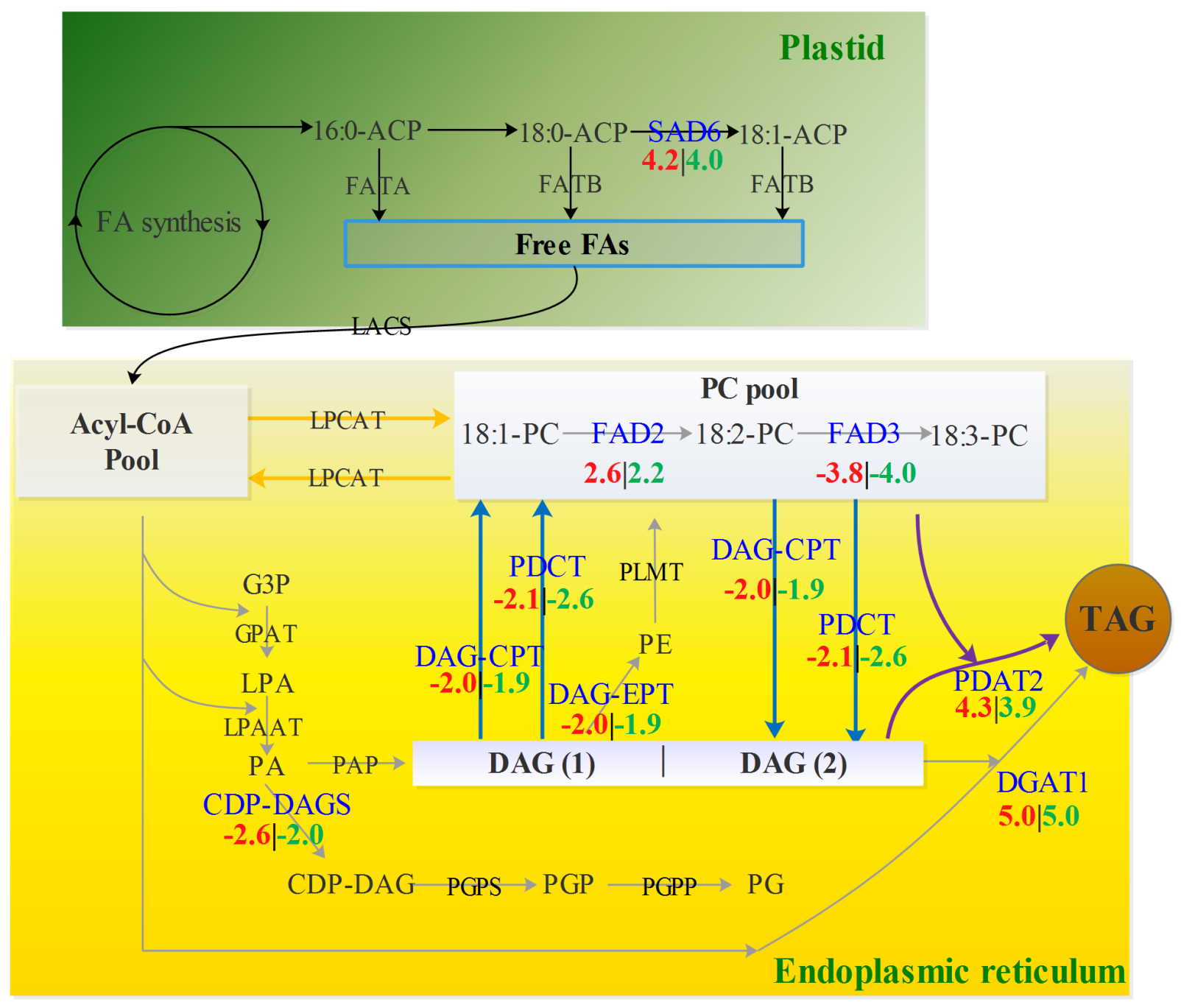

\section{Figure 5}

Transcript patterns for some key enzymes involved in lipid metabolism. Values generated from qRT-PCR indicate relative expression levels at 6 WAA from AS80 (red values) and AS-84 (green values). Those values are calculated as log2((1+E1) $\Delta C \mathrm{Ct}$ (Control-Sample)/(1+E2) $\Delta \mathrm{Ct} 2$ (Control-Sample)), E1: PCR efficiency of target-gene primer; E2: PCR efficiency of reference-gene primer; $\triangle \mathrm{Ct1}$ : the difference of $\mathrm{Ct}$ value between control and sample in experimental group; $\triangle \mathrm{Ct} 2$ : the difference of $\mathrm{Ct}$ value between control and sample in reference group. Abbreviations: fatty acid, FA; stearoyl-ACP desaturase 6, SAD6; Acyl-ACP thioesterase A, FATA; Acyl-ACP thioesterase B, FATB; long-chain Acyl-CoA synthetase LACS; lysophospholipid acyltransferase, LPCAT; phosphatidylcholine, PC; glycerol-3-phosphate, G3P; fatty acid desaturase, FAD; glycerol-3-phosphate acyltransferase, GPAT; lyso-phosphatidic acid, LPA; phosphatidic acid, PA; phosphatidic acid phosphatase, PAP; acyICoA:LPA acyltransferase, LPAAT; diacylglycerol, DAG; diacylglycerol cholinephosphotransferase, DAG-CPT; diacylglycerol ethanolaminephosphotransferase, DAG-EPT; phosphatidylcholine: diacylglycerol cholinephosphotransferase, PDCT; phospholipid:DAG acyltransferase 2, PDAT2; diacylglycerol acyltransferase 1, DGAT1; phosphatidyl-ethanolamine, PE; phosphatidyl-N-methylethanolamine N-methyltransferase, PLMT; CDP-diacylglycerol synthase, CDP-DAGS; phosphatidylglycerol-phosphate PGP; PGP synthase, PGPS; phosphatidylglycerol, PG; PG-phosphate phosphatase, PGPP. 


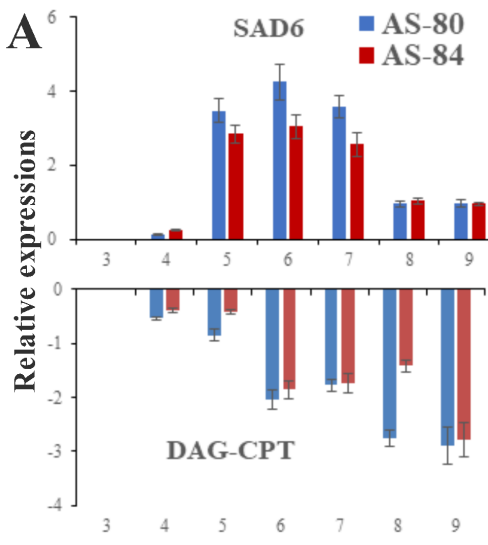

B

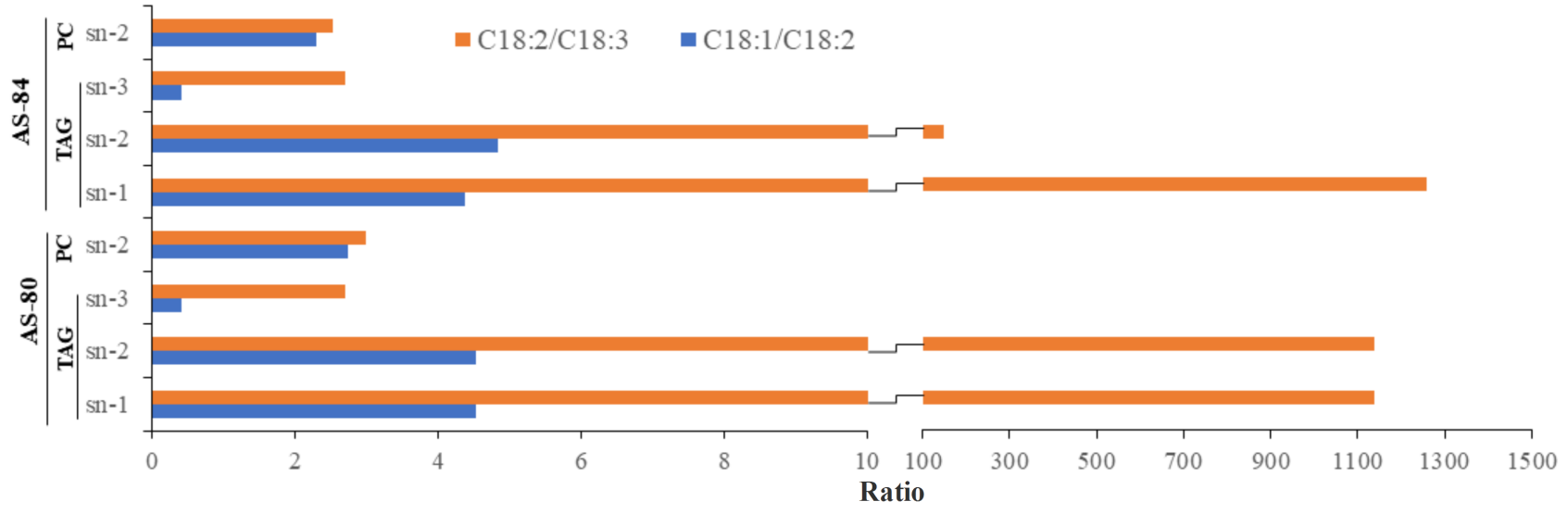

Figure 6

The calculation of relative expression levels by qRT-PCR (A) and the ratios of C18:1/ C18:2 and C18:2/ C18:3 in TAG and PC (B). Abbreviations are shown in the Fig. 5 .

\section{Supplementary Files}

This is a list of supplementary files associated with this preprint. Click to download.

- Suppledata.xlsx 